\title{
CUESTIONARIO DE AFRONTAMIENTO DEL ESTRÉS (CAE): DESARROLLO Y VALIDACIÓN PRELIMINAR
}

\author{
BONIFACIO SANDÍN Y PALOMA CHOROT \\ Universidad Nacional de Educación a Distancia
}

(Aceptado en noviembre de 2002)

\begin{abstract}
El Cuestionario de Afrontamiento del Estrés (CAE) es una medida de autoinforme diseñada para evaluar siete estilos básicos de afrontamiento: (1) focalizado en la solución del problema, (2) autofocalización negativa, (3) reevaluación positiva, (4) expresión emocional abierta, (5) evitación, (6) búsqueda de apoyo social, y (7) religión. El artículo describe el desarrollo y la validación preliminar del CAE, a partir de una muestra de estudiantes universitarios $(N=592)$. Los resultados demostraron una clara estructura factorial de siete factores que representaban los siete estilos básicos de afrontamiento. Las correlaciones entre los factores fueron bajas o moderadas. Los coeficientes de fiabilidad de Cronbach para las 7 subescalas variaron entre 0,64 y 0,92 (media $=0,79$ ). Un análisis factorial de segundo orden evidenció una estructura de dos factores, que representaban los estilos de afrontamiento racional y focalizado en la emoción. Las mujeres informaron usar más las estrategias de afrontamiento que los varones.
\end{abstract}

Palabras clave: Afrontamiento, CAE, estrés, evaluación, análisis factorial.

The Coping Strategies Questionnaire: Development and preliminary validation

The Coping Strategies Questionnaire (CAE) is a 42-item self-report measure designed to assess seven basic coping styles: (1) problem-solving coping, (2) negative auto-focused coping, (3) positive reappraisal, (4) overt emotional expression, (5) avoidance coping, (6) social support seeking, and (7) religious coping. This article describes the development and preliminary validation of the CAE, derived from a sample of college students $(\mathrm{N}=592)$. The data were analysed both by items and by sub-scales, and used exploratory factor analysis. Results showed a clear seven-factor structure involving the seven basic coping styles. Low to moderate correlations were found between the seven factors. The Cronbach's alpha reliabilities of the subscales ranged from 0.64 to 0.92 (mean $=0.79$ ). A second-order factor solution showed a two-factor structure involving rational and emotion focused coping. Results showed also that women were more likely than men to engage (to report) in most coping strategies.

Key words: Coping, CAE, stress, assessment, factor analysis.

\section{INTRODUCCIÓN}

En términos generales, el concepto de "afrontamiento» del estrés hace referencia a los esfuerzos conductuales y cognitivos que lleva a cabo el individuo para hacer frente al estrés; es decir, para tratar tanto con las demandas externas o internas generadoras del estrés, como con el

Correspondencia: Bonifacio Sandín, Facultad de Psicología, Universidad Nacional de Educación a Distancia, c/ Juan del Rosal 10 (Ciudad Universitaria), 28040 Madrid. Correo-e: bsandin@psi.uned.es. malestar psicológico que suele acompañar al estrés (Sandín, 1995). El afrontamiento del estrés es un concepto que posee un gran interés en el ámbito de la psicología clínica y la psicopatología, ya que el modo en que el individuo hace frente al estrés puede actuar como importante mediador entre las situaciones estresantes y la salud (Pelechano, 1992; Pelechano, Matud y De Miguel, 1994; Galán, Pérez San Gregorio, y Blanco, 2000; Fernández y Díaz, 2001). El afrontamiento del estrés, sin embargo, no 
constituye un concepto unitario, pues, como hemos señalado en otros lugares (p.ej., Sandín, 1995), son diversas las acepciones que se han presentado en la literatura científica. Uno de los significados del afrontamiento que ha adquirido mayor desarrollo en los últimos años tiene que ver con el concepto del estrés como proceso (Lazarus, 1966; Lazarus y Folkman, 1984; Sandín, 1995). Partiendo de esta concepción del estrés, el afrontamiento es definido como los esfuerzos en curso cognitivos y conductuales dirigidos a «manejar» las demandas internas o internas que son evaluadas como algo que grava o excede los recursos de la persona.

En esta conceptuación del afrontamiento liderada por el grupo de Lazarus se asume que el individuo utiliza diversas estrategias para tratar con el estrés, las cuales pueden ser o no adaptativas (el afrontamiento se emplea indistintamente de que el proceso del estrés sea adaptativo o desadaptativo). Si bien la consideración del afrontamiento como proceso implica asumir la dependencia contextual, el propio Lazarus (1993a,b; Lazarus y Folkman, 1984) ha defendido que las estrategias de afrontamiento pueden ser estables a través de diferentes situaciones estresantes. Este concepto del afrontamiento, por otra parte, es coherente con la idea que tenemos en psicología sobre las diferentes disposiciones personales, en el sentido que las respuestas del individuo suelen estar determinadas por una interacción entre las situaciones externas y las disposiciones personales. En este sentido, como han enfatizado Törestad, Magnuson y Oláh (1990), el afrontamiento está determinado tanto por la situación como por la persona (rasgo), lo cual sería consistente con la evidencia sobre la estabilidad del afrontamiento.

Desde este marco teórico del afrontamiento, el grupo de Lazarus ha popularizado en el ámbito de la investigación del estrés psicosocial el Ways of Coping Questionnaire (WCQ), un cuestionario de autoinforme pionero que permite obtener información sobre las estrategias de afrontamiento que emplean los individuos cuando estos se las tienen que ver con el estrés (Folkman y Lazarus, 1980, 1988; Lazarus y Folkman, 1984). La mayoría de los cuestionarios que existen actualmente sobre el afrontamiento del estrés derivan directa o indirectamente del WCQ. Aunque los resultados obtenidos con este cuestionario no siempre han dado lugar a dimensiones consistentes, Folkman y Lazarus (1988) han sugerido, como más estables, las siguientes dimensiones del WCQ: (1) confrontación, (2) distanciamiento, (3) autocontrol, (4) búsqueda de apoyo social, (5) aceptación de la responsabilidad, (6) escape-evitación, (7) planificación de solución de problemas, y (8) re-evaluación positiva. Estas 8 dimensiones fueron replicadas por Sánchez-Cánovas (1991) con la versión española del cuestionario. Los autores del cuestionario, no obstante, a veces han informado sobre otras 8 dimensiones del WCQ que no coinciden con las descritas arriba. Por ejemplo, Folkman y Lazarus (1985) han señalado las siguientes 8 subescalas del WCQ: (1) afrontamiento focalizado en el problema, (2) ensoñación o afrontamientos fantásticos ( «wishful thinking»), (3) distanciamiento, (4) enfatización de lo positivo, (5) auto-culpación, (6) reducción de la tensión, (7) auto-aislamiento, y (8) búsqueda de apoyo social (según los autores, las subescalas 2-7 se refieren a dimensiones del afrontamiento centrado en la emoción, y la subescala 8 es una dimensión mixta centrada tanto en el problema como en la emoción). Una versión española modificada del WCQ fue llevada a cabo por Rodríguez-Marín, Terol, López-Roig y Pastor (1992), la cual, a pesar de incluir sólo 36 items (el WCQ, en sus diversas formas, generalmente ha superado los 60 
items), su estructura factorial sugería 11 dimensiones básicas del afrontamiento. Aunque el WCQ ha sido el cuestionario de autoinforme sobre el afrontamiento del estrés más utilizado internacionalmente, tal vez su principal problema ha radicado en la baja consistencia factorial (como indicamos, los propios autores obtienen diferentes estructuras factoriales en diferentes estudios).

Con posterioridad al WCQ, e inspirándose de algún modo en este cuestionario, se han venido desarrollando otros cuestionarios generales del afrontamiento, tales como los de Billings y Moos (1981; Moos y Billings, 1982), Carver, Scheier y Weintraub (1989), Endler y Parker (1990), y Roger, Jarvis y Najarian (1993). Moos y Billings añadieron, a las dos dimensiones generales de Lazarus y Folkman (i.e., afrontamiento centrado en el problema y en la emoción), la dimensión de afrontamiento centrado en la evaluación. Carver et al. incrementan el número de estrategias básicas de afrontamiento. Endler y Parker añaden la dimensión de evitación como tercera dimensión general del afrontamiento. Finalmente, Roger et al. aíslan una cuarta dimensión que denominan "detachment» («desengancharse» de la situación estresante»). (Sandín, 1995; Véase Sánchez-Cánovas (1991) y DeRidder (1997), para un análisis más riguroso sobre la evaluación psicométrica de las estrategias de afrontamiento).

La Escala de Estrategias de Coping (EEC) fue desarrollada por Chorot y Sandín (1987) con la finalidad de obtener una prueba psicométrica que evaluara un amplio espectro de formas de afrontamiento del estrés. La construcción de la escala se llevó a cabo según criterios teórico-racionales, y tomando como base el cuestionario de Lazarus y Folkman (1984) y los trabajos sobre evaluación del afrontamiento de Moos y Billings (1982). Se tomó como marco teórico de referencia la organización que hacen estos últi- mos autores sobre las dimensiones generales del afrontamiento (i.e., afrontamiento centrado en la evaluación, en el problema, y en la emoción), así como las diferentes categorías asociadas a estas tres dimensiones (tres dimensiones más específicas por cada dimensión general). La EEC definitiva incluía 90 elementos que evaluaban las 9 dimensiones básicas siguientes: (1) análisis lógico (p.ej., intentar analizar las causas del problema), (2) redefinición cognitiva (p.ej., pensar que las cosas podrían ser peores), (3) evitación cognitiva (p.ej., intentar olvidarse de todo), (4) búsqueda de información o consejo (p.ej., pedir consejo a algún amigo), (5) acciones de solución de problemas (p.ej., hablar con las personas responsables), (6) desarrollo de refuerzos alternativos (p.ej., iniciar nuevos estudios), (7) regulación afectiva (p.ej., refugiarse en la lectura), (8) aceptación resignada (p.ej., aceptar las cosas como son, pues se trata de mala suerte), y (9) descarga emocional (p.ej., comportarse de forma agresiva). Estas dimensiones se agrupaban en 3 dimensiones más generales del afrontamiento centradas en (a) la evaluación cognitiva (incluía las dimensiones 1-3), (b) el problema (dimensiones 4-6), y (c) la emoción (dimensiones 7-9). Adicionalmente, y de forma no excluyente, podían obtenerse las dimensiones de evitación-escape y búsqueda de apoyo social.

Algunos de nuestros estudios llevados a cabo con la EEC sugerían que la escala poseía validez discriminante, ya que mientras ciertas formas de afrontamiento se relacionan con la salud o con tipos saludables, otras formas lo hacían con ciertos trastornos, con tipos no saludables, o con síntomas somáticos. Así, al relacionar las formas de afrontamiento del estrés con la sintomatología somática, encontramos que (a) las dimensiones de evitación, aceptación resignada, y descarga emocional correlacionaban de for- 
ma positiva y significativa con la sintomatología somática, (b) las dimensiones de solución de problemas y análisis lógico correlacionaban de forma negativa, y (c) otras dimensiones no evidenciaban correlaciones significativas (Sandín, Chorot, Segura y Pardo, 1988). En un estudio posterior en el que comparamos las estrategias de afrontamiento del estrés entre individuos con trastornos de ansiedad e individuos normales, observamos que los primeros puntuaban de forma más elevada en las dimensiones focalizadas en la emoción y en la evitación-escape, mientras que las personas normales obtenían puntuaciones más altas en las dimensiones centradas en el problema y en la evaluación (Sandín y Chorot, 1993). De forma similar, constatamos que una asociación similar se obtenía cuando relacionábamos el afrontamiento con tipos saludables y no saludables de reacción al estrés; las dimensiones de afrontamiento focalizado en la emoción y en la evitación se asociaban positivamente a los tipos no saludables y negativamente al tipo saludable, mientras que la dimensión centrada en problema se asociaba negativamente a los tipos no saludables y positivamente al tipo saludable (Sandín, Chorot, Santed y Jiménez, 1994).

Nuestros datos sobre la fiabilidad de la EEC basados en el análisis de la consistencia interna (coeficiente alfa de Cronbach) sugerían que los niveles de fiabilidad no eran uniformes a través de las 9 subescalas básicas (Chorot y Sandín, 1991). Las subescalas de búsqueda de información o consejo y evitación cognitiva poseían coeficientes alfa superiores a 0,80 , la subescala sobre el afrontamiento centrado en la consecución de refuerzos alternativos no superó el valor de $0,60, y$ las restantes subescalas poseían coeficientes que variaban entre 0,60 y 0,80 , predominando los valores por encima de 0,70 . Esto indica que al menos algunas subescalas de la EEC presentan una con- sistencia interna moderada o baja (caso de la subescala sobre refuerzos alternativos). Por otra parte, los resultados de nuestro análisis factorial de la escala no resultaron ser consistentes, ni con las nueve dimensiones establecidas a priori al construir el cuestionario, ni con las diferentes dimensiones sugeridas por el grupo de Lazarus (véase arriba) (Chorot y Sandín, 1991).

Partiendo de este análisis factorial que llevamos a cabo con datos obtenidos a partir de una muestra amplia de estudiantes universitarios, efectuamos una profunda depuración y revisión de la EEC, cuyo resultado final fue la EECRevisada (EEC-R), una escala de 54 elementos y 9 dimensiones básicas (Sandín, Valiente y Chorot, 1999). Las 9 subescalas básicas de la EEC-R incluían 6 items cada una y fueron denominadas del siguiente modo: (1) Focalizado en la situación o problema, (2) Autofocalización negativa, (3) Autocontrol, (4) Reestructuración cognitiva, (5) Expresión emocional abierta, (6) Evitación, (7) Búsqueda de apoyo social, (8) Religión, y (9) Búsqueda de apoyo profesional. En el proceso de depuración y revisión de la escala se eliminaron los items que en el análisis factorial resultaron ser hiperplanos o complejos, se redefinieron ciertos elementos para hacerlos menos ambiguos, y se incluyeron algunos nuevos items (a partir de las estrategias de afrontamiento sugeridas con mayor frecuencia por los sujetos). Así por ejemplo, se eliminaron items como "Acepté trabajos temporales ofrecidos por alguna persona conocida", "Me sentí inspirado para hacer algo creativo", y "Comencé nuevos estudios». Ejemplos de elementos redefinidos: "Le conté a familiares o amigos cómo me sentía» en lugar de "Le conté a alguien cómo me sentía», "Hice frente al problema poniendo en marcha varias soluciones concretas» en lugar de «Establecí varias soluciones concretas para hacer frente al problema». Ejemplos de 
elementos nuevos: "Acudí a la Iglesia para rogar que se solucionase el problema», «Insulté a ciertas personas».

El EEC-R ha resultado eficaz para diferenciar a los sujetos normales de los pacientes con enfermedades orgánicas (Vinaccia, Tobón, Martínez-Sánchez, y Sandín, 2001). Si bien la EEC-R supuso un cambio sustancial con respecto a su antecesora, aparte de haber sido elaborada empíricamente (a partir del análisis factorial), aún no ha sido publicado ningún trabajo que haya replicado su validez factorial. Mediante el presente estudio se pretende analizar la estructura factorial de la EEC-R partiendo de una muestra de participantes no clínicos (estudiantes universitarios). Hipotetizamos una estructura de 9 dimensiones del afrontamiento, las cuales deberían coincidir con las 9 subescalas de la prueba. Así mismo, con esta investigación se pretende proporcionar información normativa (medias y desviaciones típicas) de la escala.

\section{MÉTODO}

\section{Participantes}

La muestra de participantes estuvo constituida por 592 estudiantes universitarios procedentes de la Universidad Complutense de Madrid y de la Universidad Pública de Navarra (392 eran mujeres y 200 eran varones). El rango de edad fue de 19 a 28 años (Media $=21,32, D T=3,01$ ). Para comparar las diferencias en el uso de las estrategias de afrontamiento entre varones y mujeres se seleccionó al azar un grupo de 200 mujeres a partir de las 392 .

\section{Prueba de evaluación y procedimiento}

Los participantes cumplimentaron la EEC-R (Sandín et al., 1999), cuyas características descriptivas y psicométricas han sido indicadas arriba. La escala fue aplicada en grupos de aproximadamente 30 70 sujetos. De acuerdo con las instrucciones de la prueba, los sujetos debían indicar la frecuencia con que emplearon cada una de las 54 formas de afrontamiento indicadas, según una escala tipo Likert graduada entre 0 (Nunca) y 4 (Casi siempre), para hacer frente a las situaciones o problemas estresantes más importantes vividos durante el último año. (La prueba, no obstante, puede aplicarse, modificando las instrucciones, para evaluar el afrontamiento a una situación específica estresante). La escala incluía al final un espacio en blanco para que los participantes pudieran añadir otras formas de afrontamiento empleadas.

\section{RESULTADOS}

\section{Análisis factorial}

Los 54 elementos de la EEC-R fueron sometidos a un primer análisis factorial según el método de componentes principales y rotación oblimin (seleccionamos este método ya que asumíamos una correlación moderada entre los componentes). Para la extracción del número de factores partimos en primer lugar de nuestra asunción de 9 factores como base de la estructura simple de la EEC-R, si bien también tuvimos en cuenta otros criterios como el test de scree (Cattell, 1966), los criterios de extracción de Thurstone (1947), y los criterios de interpretabilidad de la estructura simple de Gorsuch (1983). Estos mismos criterios se aplicaron en posteriores análisis. El índice de Kaiser-Meyer-Olkin $(=0,89)$ sugería que los datos eran adecuados para el cálculo del análisis factorial.

En este primer análisis, si bien el test de scree sugería una estructura de 8 factores, consideramos necesario llevar a cabo una extracción de 9 factores con 
objeto de contrastar las 9 dimensiones de la escala. El análisis sugería un factor residual (noveno factor), y ocho factores que representaban 8 de las 9 dimensiones de la escala (no aparecía un factor relativo a la dimensión de autocontrol; los elementos de esta subescala saturaban en varios factores). Tras este análisis, se eliminaron los 6 items correspondientes a la subescala de autocontrol, así como también los items 6 [«Me alejé del problema temporalmente (tomando unas vacaciones, descansando, viajando, etc.)", dimensión de evitación], 16 ( «Tuve fe en que ocurriese algún milagro», dimensión de religión) y 53 («Lloré», dimensión de expresión emocional abierta). Estos tres items se eliminaron por ser hiperplanos o complejos, y fueron sustituidos por otros tres (a) obtenidos entre los incorporados con mayor frecuencia por los participantes y (b) considerando su coherencia con la dimensión correspondiente. De este modo se incluyeron los items 5,14 y 39 del nuevo cuestionario (véase el Anexo I). También se eliminaron los 6 items de la subescala de búsqueda de ayuda profesional, ya que, aunque configuraban un claro factor, la frecuencia con que los participantes empleaban las estrategias de esta dimensión resultó ser muy baja (como media de las 6 estrategias menos del $80 \%$ de los sujetos). Así pues, procedimos a un segundo análisis partiendo de los 42 elementos restantes de la escala.

Aplicando los criterios de extracción indicados arriba, obtuvimos 7 claros factores que explicaban el $55,3 \%$ de la varianza total. Los autovalores iniciales correspondientes a estos 7 factores fueron, respectivamente, $8,3(20,3 \%$ de la varianza), 4,6 (11,2\%), 3,2 (7,8\%), 2,2 $(5,3 \%), 1,9(4,7 \%), 1,4(3,2 \%)$ y 1,2 $(2,8 \%)$. En la Tabla 1 indicamos los 7 factores del afrontamiento y las saturaciones correspondientes a cada uno de los 42 elementos del cuestionario después de la rotación (oblimin). Partiendo de que el número de items es elevado, y puesto que la estructura factorial resultante tras la rotación ha resultado ser muy clara y robusta (sólo dos elementos saturaron en una dimensión adicional, i.e. los elementos 17 y 22), para cada ítem únicamente indicamos en la tabla las saturaciones relacionadas con su dimensión específica. Puede observarse que los 7 factores corresponden a las siete dimensiones básicas del afrontamiento que evalúa el cuestionario, i.e., Factor 1: búsqueda de apoyo social (BAS), Factor 2: expresión emocional abierta (EEA), Factor 3: religión (RLG), Factor 4: focalizado en la solución del problema (FSP), Factor 5: evitación (EVT), Factor 6: auto-focalización negativa (AFN), y Factor 7: re-evaluación positiva (REP). El cuestionario resultante tras los respectivos análisis factoriales ha sido denominado Cuestionario de Afrontamiento del Estrés (CAE; Véanse la Tabla 1 y el Anexo I).

En la Tabla 2 indicamos las correlaciones entre los factores (correlaciones basadas en las puntuaciones factoriales), oscilando éstas entre 0,40 (BAS-FSP) y $-0,03$ (FSP-AFN). En general, y como cabría esperar, las correlaciones son moderadas o bajas, lo cual confiere validez a la relativa independencia entre las dimensiones. Las correlaciones más altas se dan con el factor Focalizado en la solución del problema, el cual correlaciona de forma moderada con Búsqueda de apoyo social y Reevaluación positiva.

Con objeto de examinar la existencia de posibles dimensiones más generales del CAE, llevamos a cabo un análisis factorial de orden superior basándonos en las puntuaciones factoriales obtenidas tras la rotación oblicua de la estructura de orden inferior de 7 factores. Los resultados de este análisis están indicados en la Tabla 3. La estructura factorial resultante comprendía dos factores que explicaban el $49,3 \%$ de la varianza total (los autovalo- 
Tabla 1. Factores (subescalas) del CAE, con indicación de los pesos factoriales (rotación oblimin) y las correlaciones corregidas ítem-subescala $(N=592)$

\begin{tabular}{ccc}
\hline Ítem del CAE & Peso factorial & $\begin{array}{c}\text { Correlación } \\
\text { ítem-subescala }\end{array}$ \\
\hline
\end{tabular}

Factor 1: Búsqueda de apoyo social (BAS)

6. Contar los sentimientos a familiares o amigos

0,77

0,69

13. Pedir consejo a parientes o amigos

0,78

0,75

20. Pedir información a parientes o amigos

27. Hablar con amigos o parientes para ser tranquilizado

0,85

0,80

34. Pedir orientación sobre el mejor camino a seguir

0,84

0,81

41. Expresar los sentimientos a familiares o amigos

0,86

0,81

0,80

0,76

Factor 2: Expresión emocional abierta (EEA)
4. Descargar el mal humor con los demás
11. Insultar a otras personas
18. Comportarse hostilmente
25. Agredir a alguien
32. Irritarse con la gente
39. Luchar y desahogarse emocionalmente

0,64

0,64

0,52

0.76

0,50

0,71

0,61

0,73

0,43

0,56

0,60

Factor 3: Religión (RLG)

7. Asistir a la Iglesia

$\begin{array}{ll}0,73 & 0,58 \\ 0,52 & 0,49 \\ 0,86 & 0,75 \\ 0,76 & 0,70 \\ 0,83 & 0,73 \\ 0,87 & 0,73\end{array}$

14. Pedir ayuda espiritual

21. Acudir a la Iglesia para rogar se solucione el problema

28. Confiar en que Dios remediase el problema

35. Rezar

0,87

0,46

42. Acudir a la Iglesia para poner velas o rezar

1. Analizar las causas del problema

8. Seguir unos pasos concretos

0,86

22. Hablar con las personas implicadas ${ }^{1}$

29. Poner en acción soluciones concretas

36. Pensar detenidamente los pasos a seguir

0,33

0,72

0,76

0,48

0,74

0,70

0,73

Factor 5: Evitación (EVT)

5. Concentrarse en otras cosas

12. Volcarse en el trabajo $u$ otras actividades

0,62

0,48

19. "Salir» para olvidarse del problema

26. No pensar en el problema

0,70

0,57

0,75

0,60

$0,64 \quad 0,47$

33. Practicar deporte para olvidar

0,76

0,48

40. Tratar de olvidarse de todo

0,63

0,52

Factor 6: Autofocalización negativa (AFN)
2. Autoconvencerse negativamente
0,45
0,30
9. No hacer nada ya que las cosas suelen ser malas
0,47
0,33
16. Autoculpación
0,34
0,27
23. Sentir indefensión respecto al problema
0,77
0,52
30. Asumir la propia incapacidad para resolver la situación
0,63
0,45
37. Resignarse
0,48
0,35

Factor 7: Reevaluación positiva (REP)

3. Ver los aspectos positivos

$0,67 \quad 0,55$

10. Sacar algo positivo de la situación

0,70

0,56

17. Descubrir que en la vida hay gente buena ${ }^{1}$

0,32

0,33

24. Comprender que hay cosas más importantes

0,60

0,45

31. No hay mal que por bien no venga

0,66

0,48

38. Pensar que el problema pudo haber sido peor

0,29

0,28

Nota. Los enunciados de los items aparecen de forma abreviada (véase el Anexo I). (1) Los items 17 y 22 también saturaron $(\geq 0,30)$ en el Factor 1 . 
res de los restantes factores eran inferiores a la unidad). Basándonos en las saturaciones que poseen los diferentes factores primarios en los factores de orden superior, parece claro que el primer factor corresponde a una dimensión relacionada fundamentalmente con el problema o la situación estresante, y con componentes más bien lógicos o racionales, mientras que el segundo factor representaría más bien los componentes emocionales del afrontamiento. La correlación entre ambos factores fue de $-0,20$. ciones en función de los items y las subescalas, en general las correlaciones tienden a ser entre moderadas y altas, lo que sugiere que los elementos representan apropiadamente a las subescalas correspondientes (fenómeno este apoyado también por los resultados que derivan del análisis factorial). Las correlaciones más altas corresponden a la subescala BAS (correlación media $=0,77$ ), siendo las más bajas las relativas a la subescala AFN (correlación media $=0,37$ ). Las correlaciones medias pertenecientes a las restantes subescalas

Tabla 2. Correlaciones entre los factores y coeficientes alfa de Cronbach

\begin{tabular}{llcccccccc}
\hline & & BAS (1) & EEA (2) & RLG (3) & FSP (4) & EVT (5) & AFN (6) & REP (7) \\
\hline BAS & (Factor 1) & $(0,92)$ & & & & & & & \\
EEA & (Factor 2) & 0,06 & $(0,74)$ & & & & & \\
RLG & (Factor 3) & $\mathbf{0 , 1 8}$ & $\mathbf{0 , 0 9}$ & $(0,86)$ & & & & \\
FSP & (Factor 4) & 0,40 & $\mathbf{0 , 0 2}$ & 0,01 & $(0,85)$ & & & \\
EVT & (Factor 5) & 0,23 & $\mathbf{0 , 2 0}$ & $\mathbf{0 , 1 4}$ & 0,12 & $(0,76)$ & & \\
AFN & (Factor 6) & 0,07 & $\mathbf{0 , 3 4}$ & $\mathbf{0 , 1 9}$ & $-0,03$ & $\mathbf{0 , 2 9}$ & $(0,64)$ & \\
REP & (Factor 7) & 0,26 & $\mathbf{0 , 1 0}$ & $\mathbf{0 , 1 1}$ & 0,38 & $\mathbf{0 , 2 4}$ & $\mathbf{0 , 1 4}$ & $(0,71)$ \\
\hline
\end{tabular}

Nota. Entre paréntesis se indican los coeficientes alfa correspondientes a las 7 subescalas. BAS = Búsqueda de apoyo social, EEA = Expresión emocional abierta, RLG = Religión, FSP = Focalizado en la solución del problema, EVT $=$ Evitación, $A F N=$ Autofocalización negativa, $R E P=$ Reevaluación positiva.

\section{Fiabilidad}

La fiabilidad (consistencia interna) del CAE la hemos analizado a través del coeficiente alfa de Cronbach y de las correlaciones corregidas ítem-subescala. En lo que concierte a los coeficientes alfa (véase la Tabla 2), las subescalas BAS, RLG y FSP presentan coeficientes $\geq 0,85$ (niveles excelentes), los subescalas EEA, EVT y EEP presentan coeficientes entre 0,71 y 0,76 , correspondiendo el coeficiente más bajo $(0,64)$ a la subescala ANF. Considerados conjuntamente, podríamos decir que el CAE presenta una fiabilidad entre excelente y aceptable, sobre todo si tenemos en cuenta que cada subescala posee sólo 6 elementos.

Las correlaciones corregidas ítem-subescala (excluido el ítem) están indicadas en la Tabla 1. Aun cuando existen notables diferencias en la magnitud de las correla- varían entre 0,44 (subescala REP) y 0,67 (subescala RLG).

\section{Datos normativos}

Las medias y desviaciones típicas relativas a las 7 subescalas del CAE se indican en la Tabla 3. Puede apreciarse que las puntuaciones más elevadas corresponden a las subescalas REP, FSP y BAS, lo que sugiere que los sujetos suelen emplear con mayor frecuencia estas formas de afrontamiento. RLG, EEA y AFN son las subescalas que presentan las puntuaciones medias más bajas.

Para analizar las posibles diferencias entre los varones y las mujeres calculamos un análisis de varianza multivariado de un factor (sexo) y 7 variables dependientes (las 7 subescalas del CAE). Los resultados indicaron la exis- 
Tabla 3. Análisis de componentes principales de orden superior de la estructura del CAE: Saturaciones en los factores de orden superior $(N=592)$

\begin{tabular}{lrr}
\hline \multicolumn{1}{c}{ Factores primarios del CAE } & Factor 1 & Factor 2 \\
\hline Factor 1: Búsqueda de apoyo social (BAS) & $\mathbf{0 , 7 2}$ & 0,06 \\
Factor 2: Expresión emocional abierta (EEA) & $-\mathbf{0 , 1 1}$ & $\mathbf{0 , 7 0}$ \\
Factor 3: Religión (RLG) & $-\mathbf{0 , 0 7}$ & $\mathbf{- 0 , 4 3}$ \\
Factor 4: Focalizado en la solución del problema (FSP) & $\mathbf{0 , 8 4}$ & $-0,19$ \\
Factor 5: Evitación (EVT) & $-0,26$ & $\mathbf{- 0 , 5 6}$ \\
Factor 6: Autofocalizací́n negativa (AFN) & $-0,11$ & $\mathbf{0 , 8 0}$ \\
Factor 7: Reevaluación positiva (REP) & $\mathbf{0 , 6 6}$ & $\mathbf{0 , 1 4}$ \\
Autovalor & & \\
\% de varianza total explicada & 2,05 & 1,40 \\
\hline
\end{tabular}

Nota. En negrita se indican las saturaciones $\geq 0,30$.

Tabla 4. Medias y desviaciones típicas (entre paréntesis) obtenidas en las diferentes subescalas del CAE en función de los grupos de varones $(N=200)$ y mujeres $(N=200)$

\begin{tabular}{lrrrc}
\hline & \multicolumn{1}{c}{ Total } & \multicolumn{1}{c}{ Varones } & Mujeres & \\
\cline { 2 - 5 } \multicolumn{1}{c}{ CAE (subescalas) } & \multicolumn{1}{c}{ Media $(D T)$} & \multicolumn{1}{c}{ Media $(D T)$} & Media $(D T)$ & $F(1,398)$ \\
\hline Búsqueda de apoyo social (BAS) & $12,87(6,46)$ & $11,34(6,35)$ & $14,40(6,21)$ & $23,6^{\star \star}$ \\
Expresión emocional abierta (EEA) & $6,14(3,65)$ & $6,07(3,69)$ & $6,22(3,62)$ & 0,2 \\
Religión (RLG) & $5,76(5,37)$ & $5,09(4,74)$ & $6,44(5,86)$ & $6,4^{\star}$ \\
Focalizado en la solución del problema (FSP) & $13,58(5,09)$ & $12,60(5,16)$ & $14,55(4,83)$ & $15,3^{\star \star}$ \\
Evitación (EVT) & $9,16(4,48)$ & $8,74(4,53)$ & $9,58(4,39)$ & 3,5 \\
Autofocalización negativa (AFN) & $6,22(3,54)$ & $6,17(3,52)$ & $6,27(3,54)$ & 0,1 \\
Reevaluación positiva (REP) & $13,90(4,06)$ & $12,87(3,94)$ & $14,93(3,92)$ & $27,3^{\star \star}$ \\
\hline
\end{tabular}

Nota. Rango de puntuaciones para todas las subescalas $=0-24$. La prueba de $F$ se refiere a las diferencias entre varones y mujeres.

${ }^{\star} p<0,05,{ }^{\star \star} p<0,001$ (se aplicó la corrección de Bonferroni).

tencia de efectos significativos del factor sexo [Hotelling $F(7,392)=5,9, p<$ $0,001]$. Posteriormente se calcularon los efectos univariados de la variable sexo para cada una de las variables dependientes (en la Tabla 3 indicamos cada prueba de $F$ y su significación estadística). Como podemos observar, las mujeres obtienen puntuaciones más elevadas que los varones en las subescalas de afrontamiento BAS, RLG, FSP, y REP, no existiendo diferencias significativas en las restantes dimensiones. En ninguna subescala de afrontamiento alcanzan los varones puntuaciones más elevadas que las mujeres.

\section{DISCUSIÓN}

El presente estudio tenía como finalidad examinar la estructura factorial del EEC$\mathrm{R}$, así como también proporcionar evidencia sobre su fiabilidad y normas (medias y desviaciones típicas). No obstante, el estudio ha supuesto una profunda revisión de la prueba, cuyo resultado final ha sido un cuestionario más breve y consistente que hemos denominado Cuestionario de Afrontamiento del Estrés (CAE) (se ha modificado el nombre del cuestionario debido a que actualmente está consolidada la denominación de "afrontamiento» como equivalente al término anglosajón de «coping», fenómeno 
que no era evidente cuando desarrollamos, en 1987, la primera forma de la prueba, denominada «Escala de Estrategias de Coping»).

Nuestro análisis sugiere que el CAE posee una estructura factorial robusta que consiste en 7 factores primarios o dimensiones básicas del afrontamiento. En términos generales, la mayoría de los factores obtenidos son consistentes con las dimensiones que se han venido señalando en la literatura sobre las estrategias del afrontamiento, tales como la búsqueda de apoyo social, la evitación, la focalización en la solución del problema, la reevaluación positiva, la religión, y la descarga emocional (nosotros la hemos definido como "expresión emocional abierta») (Folkman y Lazarus, 1980, 1985, 1988; Moos y Billings, 1982; Lazarus y Folkman, 1984; Sandín, 1989, 1995; Carver et al., 1989; Rodríguez-Marín, 1995). Sin embargo, una dimensión primaria del afrontamiento que no había sido señalada en la literatura es la que hemos denominado como "autofocalización negativa». En nuestros primeros estudios con el EEC (Sandín et al., 1988; Chorot y Sandín, 1991) no encontramos apoyo empírico para algunas conocidas dimensiones del afrontamiento de carácter negativo como la autoculpa, la negación, la resignación (o aceptación resignada), y el autoaislamiento. Más bien, lo que encontramos fue que algunos de los elementos que representaban a estas variables solían saturar en una misma dimensión del afrontamiento, dimensión que hemos denominado como autofocalización negativa por el carácter negativo que es común a todas ellas. Esta dimensión fue incorporada al EEC-R y ha sido replicada en el presente estudio.

En la literatura científica sobre las dimensiones primarias del afrontamiento no es fácil encontrar estabilidad de los factores a través de diferentes estudios, bien con el mismo cuestionario, o bien con cuestionarios similares. Nuestros resultados sobre la estructura del afrontamiento sugieren que las 7 dimensiones aisladas son bastante consistentes y confieren validez interna a las 7 subescalas del CAE. Por otra parte, dado que las correlaciones entre los factores son bajas o moderadas, resulta apropiado el empleo de estas dimensiones para evaluar psicométricamente las formas básicas del afrontamiento. Otros cuestionarios clásicos (p.ej., Billings y Moos, 1981; Folkman y Lazarus, 1988; Carver et al., 1989) suelen incluir otras muchas (y dispares) dimensiones primarias del afrontamiento (p.ej., autocontrol, distracción, análisis lógico, aceptación resignada, represión emocional, etc.). Sin embargo, muchas de estas dimensiones no han podido ser confirmadas de forma consistente a través de los diferentes estudios.

Aunque pensamos que los 7 factores primarios pueden utilizarse adecuadamente para evaluar las 7 dimensiones básicas del afrontamiento, existe evidencia de que éste puede entenderse también según dimensiones más generales. Nuestro análisis factorial de orden superior dio lugar a dos factores. El primer factor es equivalente a la clásica dimensión de afrontamiento que ha venido recibiendo en la literatura diversas denominaciones, tales como afrontamiento racional (Roger et al., 1993), afrontamiento focalizado en el problema (Folkman y Lazarus, 1980, 1988), afrontamiento activo (Billings y Moos, 1981), y afrontamiento focalizado en la tarea (Endler y Parker, 1990). La segunda dimensión coincide, así mismo, con la dimensión general de afrontamiento centrada en la emoción (Folkman y Lazarus, 1980, 1988; Endler y Parker, 1990; Roger et al., 1993) o con el afrontamiento pasivo (Billings y Moos, 1981). Posiblemente estas sean las dos dimen- 
siones generales más consistentes del afrontamiento, las cuales, aunque no coinciden, guardan cierta similitud con estilos clásicos de afrontamiento del estrés (p.ej., represión vs. sensibilización, atenuación vs. incremento, rechazo vs. atención, pasivo vs. activo, etc.; véase Sandín, 1989).

Si bien el segundo factor parece representar una dimensión centrada en la emoción (autofocalización negativa y expresión emocional), dos dimensiones primarias (evitación y religión) saturan de forma elevada en este factor pero de forma negativa. La evitación como forma general de afrontamiento ha sido sugerida recientemente por varios autores que han factorizado cuestionarios sobre estrategias de afrontamiento similares al CAE (Endler y Parker, 1990; Roger et al., 1993; Lyne y Roger, 2000). En nuestro estudio no emerge esta dimensión tras el análisis de segundo orden (aunque sí en el análisis de primer orden). Finalmente, algo similar ocurre con la dimensión de afrontamiento focalizado en la religión: ha sido considerada como una dimensión general de afrontamiento (Pargament, Koenig y Perez, 2000) y satura de forma negativa en nuestra dimensión de afrontamiento emocional.

Dado que las 7 dimensiones primarias del CAE parecen bastante consistentes, tanto desde el punto de vista factorial (i.e., magnitud de las saturaciones factoriales) como desde la evidencia que proporcionan los análisis de fiabilidad (coeficientes alfa y correlaciones ítem-subescala), es recomendable la utilización de las 7 subescalas del CAE para evaluar las estrategias de afrontamiento del estrés. Adicionalmente, si se desean medidas más generales del afrontamiento, podrían obtenerse las subescalas de afrontamiento racional (con predomino de focalización en el problema) (subescalas FSP, REP y BAS) y afrontamiento emocional (subescalas AFN y EEA). Los coeficientes alfa de estas dos subescalas generales fueron 0,91 y 0,79 , respectivamente.

Uno de los objetivos de este estudio consistió en proporcionar información normativa del cuestionario (medias y desviaciones típicas). Tras comparar las puntuaciones medias entre los grupos de varones y mujeres constatamos que las mujeres obtenían valores significativamente más elevados que los hombres en las formas de afrontamiento centradas en la búsqueda de apoyo social, la religión, la solución del problema, y la reevaluación positiva (los varones no obtuvieron puntuaciones superiores a las mujeres en ninguna de las 7 dimensiones). Estos resultados están de acuerdo con la evidencia sobre las diferencias sexuales en el uso de estrategias de afrontamiento, evidencia que indica de forma consistente que las mujeres emplean en general más que los hombres la mayoría de las estrategias de afrontamiento (véase el estudio de metaanálisis de Tamres, Janicki y Helgeson, 2002).

En resumen, el presente estudio presenta la nueva prueba de autoinforme del afrontamiento denominada Cuestionario de Afrontamiento del Estrés (CAE), forma revisada de la EEC-R. Presentamos información sobre su desarrollo y el marco teórico en el que se apoya, así como evidencia empírica sobre su estructura factorial, fiabilidad, medias y desviaciones típicas. La escala está constituida por 7 subescalas bastante consistentes factorialmente. Aunque el cuestionario está primariamente dirigido a evaluar el afrontamiento como rasgo, modificando las instrucciones puede emplearse para evaluar el afrontamiento ante situaciones o momentos específicos de estrés. Futuros estudios con el cuestionario deberán confirmar y extender los datos que hemos presentado sobre su fiabilidad y validez, así como su capacidad para actuar como predictor o mediador de la influencia del estrés sobre la salud. 


\section{REFERENCIAS BIBLIOGRÁFICAS}

Billings, A.G., y Moos, R.H. (1981). The role of coping responses and social resources in attenuating the stress of life events. Journal of Behavioral Medicine, 4, 139-157.

Carver, C.S., Scheier, M.F., y Weintraub, J.J. (1989). Assessing coping strategies: A theoretically based approach. Journal of Personality and social Psychology, 56, 267283.

Cattell, R.B. (1966). The scree test for the number of factors. Multivariate Behavioral Research, 3, 245-276.

Chorot, P. y Sandín, B. (1991). Escala de Estrategias de Coping (EEC). III Congreso de Evaluación Psicológica. Barcelona, 2528 de septiembre, p. 337 (Actas de Congreso).

Chorot, P., y Sandín, B. (1987). Escala de Estrategias de Coping (EEC). Madrid: Universidad Nacional de Educación a Distancia. (Policopiado).

DeRidder, D. (1997). What is wrong with coping assessment? A review of conceptual and methodological issues. Psychology \& Health, 12, 417-431.

Endler, N.S., y Parker, D.A. (1990). Multidimensional assessment of coping: A critical evaluation. Personality and Individual Differences, 58, 844-854.

Fernández, M.D., y Díaz, M.A. (2001). Relación entre estrategias de afrontamiento, síndromes clínicos y trastornos de personalidad en pacientes esquizofrénicos crónicos. Revista de Psicopatología y Psicología Clínica, 6, 129-136.

Folkman, S., y Lazarus, R.S. (1980). An analysis of coping in a middle-aged community sample. Journal of Health and Social Behavior, 21, 219-239.

Folkman, S., y Lazarus, R.S. (1985). If it changes it must be a process: Study of emotion and coping during three stages of a college examination. Journal of Personality and Social Psychology, 48, 150-170.

Folkman, S., y Lazarus, R.S. (1988). Manual for the Ways of coping questionnaire. Palo Alto, CA: Consulting Psychologists Press.

Galán, A., Pérez San Gregorio, M.A., y Blanco, A. (2000). Análisis del uso de estrate- gias de afrontamiento en la enfermedad pulmonar obstructiva crónica (EPOC): Implicaciones conceptuales. Revista de Psicopatología y Psicología Clínica, 5, 179190.

Gorsuch, R. (1983). Factor analysis. Hillsdale, NJ: Erlbaum.

Lazarus, R.S. (1966). Psychological stress and the coping process. New York: MaCrawHill.

Lazarus, R.S. (1993a). Coping theory and research: Past, present, and future. Psychosomatic Medicine, 55, 234-247.

Lazarus, R.S. (1993b). From psychological stress to the emotions: A history of changing outlooks. Annual Review of Psychology, 44, 1-21.

Lazarus, R.S. y Folkman, S. (1984). Stress, appraisal and coping. New York: Springer Publishing Company.

Lyne, K., y Roger, D. (2000). A psychometric re-assessment of the COPE questionnaire. Personality and Individual Differences, 29, 321-335.

Moos, R.H., y Billings, A.G. (1982). Conceptualizing and measuring coping resources and processes. En L. Goldberger y S. Breznitz (Eds.), Handbook of stress: Theoretical and clinical aspects (pp. 212-230). New York: Free Press.

Pargament, K.I., Koenig, H.G., y Perez, L.M. (2000). The many methods of religious coping: Development and initial validation of the RCOPE. Journal of clinical Psychology, 56, 519-543.

Pelechano, V. (1992). Personalidad y estrategias de afrontamiento en pacientes crónicos. Análisis y Modificación de Conducta, 18, 167-202.

Pelechano, V., Matud, P., y De Miguel, A. (1994). Estrés, personalidad y salud: Un modelo no sexista del estrés. Valencia: Alfaplús.

Rodríguez-Marín, J. (1995). Psicología social de la salud. Madrid: Síntesis.

Rodríguez-Marín, J., Terol, M.C., López-Roig, S., y Pastor, M.A. (1992). Evaluación del afrontamiento al estrés: Propiedades psicométricas del Cuestionario de formas de Afrontamiento de Acontecimientos Estresantes. Revista de Psicologia de la Salud, 4, 59-84. 
Roger, D., Jarvis, G., y Najarian, B. (1993). Detachment and coping: The construction and validation of a new scale for measuring coping strategies. Personality and Individual Differences, 15, 619-626.

Sánchez-Cánovas, J. (1991). Evaluación de las estrategias de afrontamiento. En Buela, G. y Caballo, V. E. (Eds.), Manual de psicología clínica aplicada (pp. 247-270). Madrid: Siglo XXI.

Sandín, B. (1989). Estrés, coping y alteraciones psicofisiológicas. En B. Sandín y J. Bermúdez (Eds.), Procesos emocionales y salud (pp. 45-72). Madrid: UNED.

Sandín, B. (1995). El estrés. En A. Belloch, B. Sandín y F. Ramos (Eds.). Manual de psicopatología, Vol. 2 (pp. 3-52). Madrid: McGraw-Hill.

Sandín, B., y Chorot, P. (1993). Stress and anxiety: Diagnosis validity of anxiety disorders according to life events stress, ways of coping and physical symptoms. Psiquis, 14, 178-184.

Sandín, B., Chorot, P. Segura, A. y Pardo, A. (1988). Estrés, coping y síntomas somáticos. IV Congreso de la Asociación Española de Terapia del Comportamiento. Gandía, 10-12 de marzo.
Sandín, B., Chorot, P., Santed, M.A., y Jiménez, M.P. (1994). Stress behavior types, personality, alexithymia, coping and statetrait anger expression. Stress News, 5(4), 2-8.

Sandín, B., Valiente, R.M., y Chorot, P. (1999). Evaluación del estrés psicosocial. En B. Sandín (Ed.), El estrés psicosocial: Conceptos y consecuencias clínicas (pp. 245316). Madrid: UNED-FUE.

Tamres, L.K., Janicki, D., y Helgeson, V.S. (2002). Sex differences in coping behavior: A meta-analytic review and an examination of relative coping. Personality and social Psychology Review, 6, 2-30.

Thurstone, L.L. (1947). Multiple factor analysis. Chicago: University of Chicago Press.

Törestad, B., Magnusson, D., y Oláh, A. (1990). Coping, control, and experience of anxiety: An interaccional perspective. Anxiety Research, 3, 1-16.

Vinaccia, S., Tobón, S., Martínez, F., y Sandín, B. (2001). Estrés y úlcera péptica duodenal en sujetos infectados con helicobacter pylori: Un estudio controlado. Revista Internacional de Psicología Clínica y de la Salud, 1, 317-331. 


\section{ANEXO I \\ CAE \\ Cuestionario de Afrontamiento del Estrés}

Nombre

Edad

Sexo

Instrucciones: En las páginas que siguen se describen formas de pensar y comportarse que la gente suele emplear para afrontar los problemas o situaciones estresantes que ocurren en la vida. Las formas de afrontamiento descritas no son ni buenas ni malas, ni tampoco unas son mejores o peores que otras. Simplemente ciertas personas utilizan unas formas más que otras. Para contestar debe leer con detenimiento cada una de las formas de afrontamiento y recordar en qué medida Vd. la ha utilizado recientemente cuando ha tenido que hacer frente a situaciones de estrés. Hodee con un cfrculo el número que mejor represente el grado en que empleó cada una de las formas de afrontamiento del estrés que se indican. Aunque este cuestionario a veces hace referencia a una situación o problema, tenga en cuenta que esto no quiere decir que Vd. piense en un único acontecimiento, sino más bien en las situaciones o problemas más estresantes vividos recientemente (aproximadamente durante el pasado año).

\begin{tabular}{c|c|c|c|c|}
0 & 1 & 2 & 3 & 4 \\
\hline Nunca & Pocas veces & A veces & Frecuentemente & Casi siempre
\end{tabular}

\section{¿Cómo se ha comportado habitualmente ante situaciones de estrés?}

1. Traté de analizar las causas del problema para poder hacerle frente...

$\begin{array}{lllll}0 & 1 & 2 & 3 & 4\end{array}$

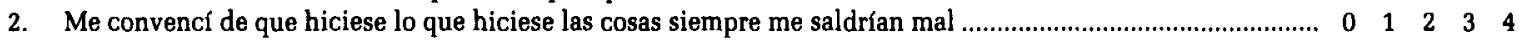

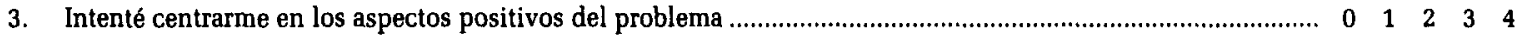

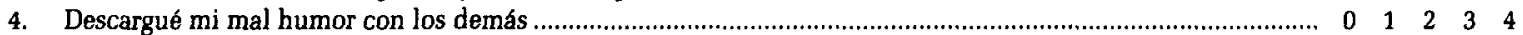

5. Cuando me venía a la cabeza el problema, trataba de concentrarme en otras cosas ........................................ 0

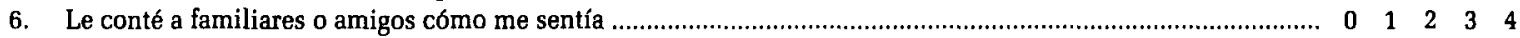

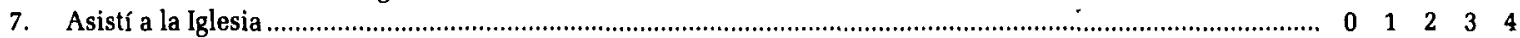

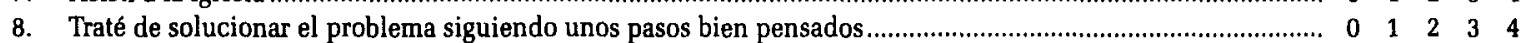

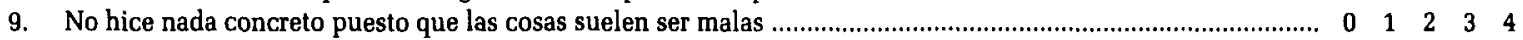

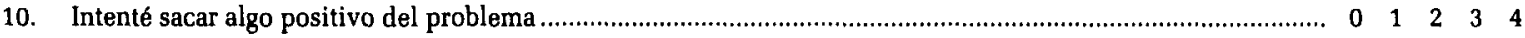

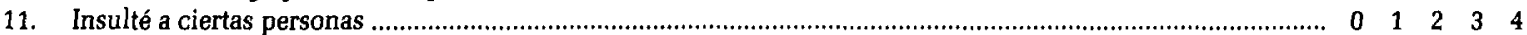

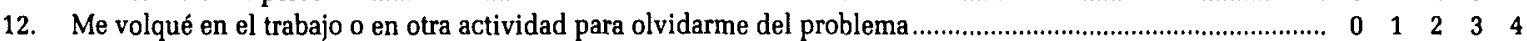

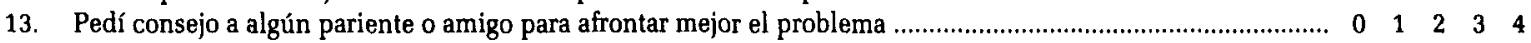

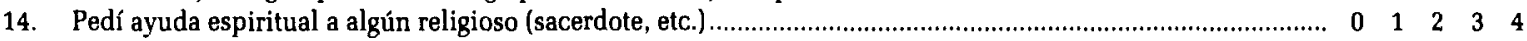

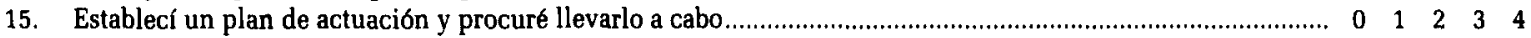

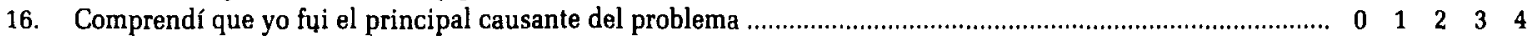

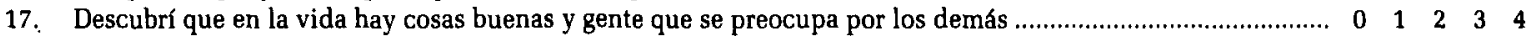

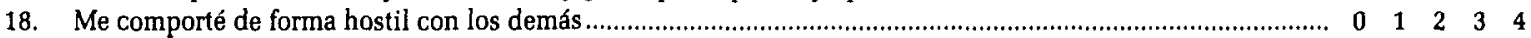

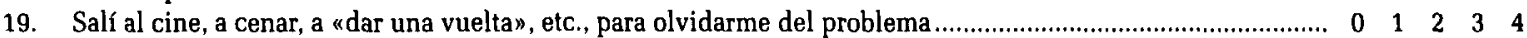

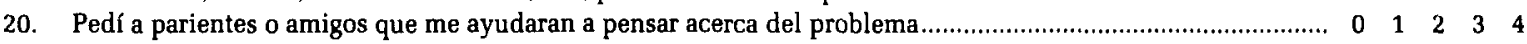

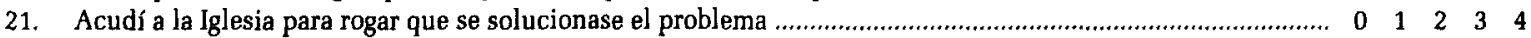

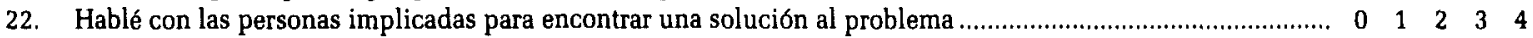

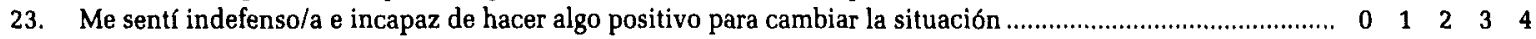

24. Comprendí que otras cosas, diferentes del problema, eran para mí más importantes .................................. 0

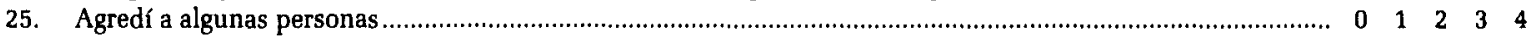

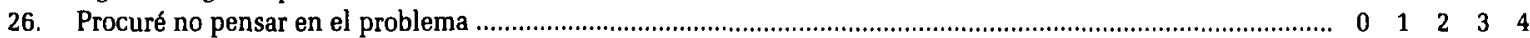

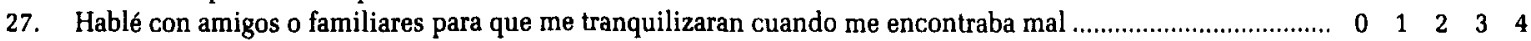

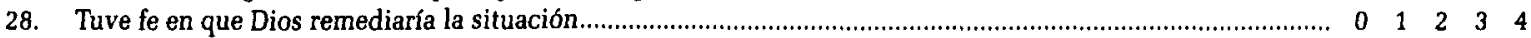

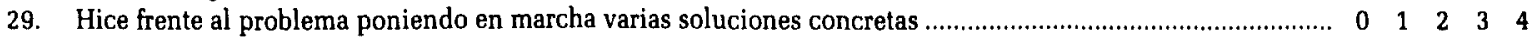

30. Me di cuenta de que por mí mismo no podía hacer nada para resolver el problema ...................................... 0

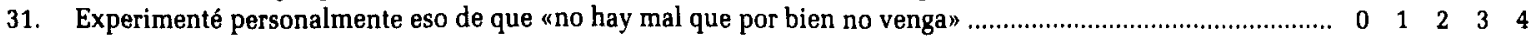

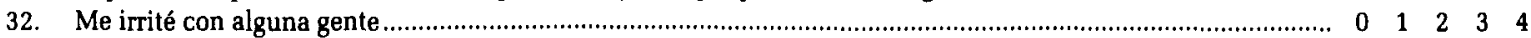

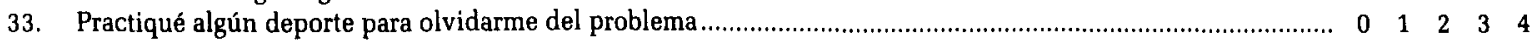

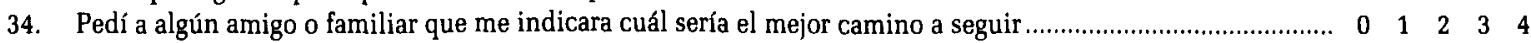

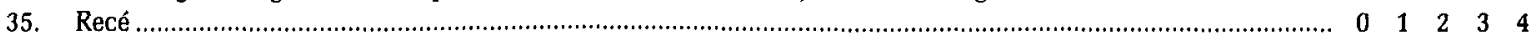

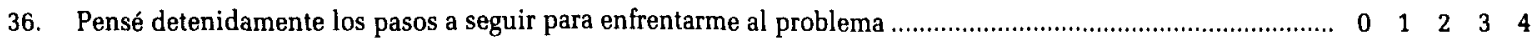

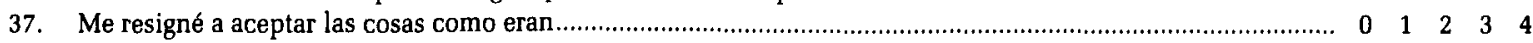

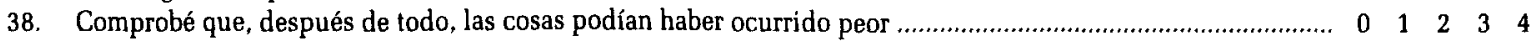

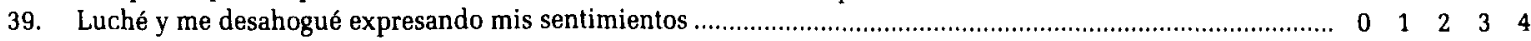

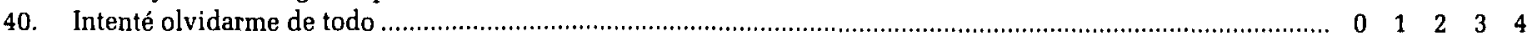

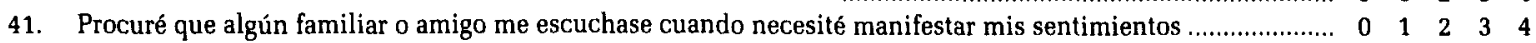

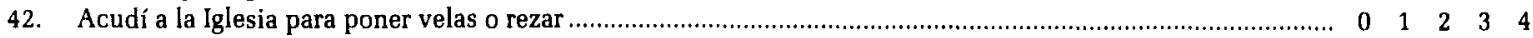


Corrección de la prueba

Se suman los valores marcados en cada ítem, según las siguientes subescalas:

Focalizado en la solución del problema (FSP):

Autofocalización negativa (AFN):

$1,8,15,22,29,36$.

Reevaluación positiva (REP):

2 , 9, 16, 23, 30, 37 .

Expresión emocional abierta (EEA):

$3,10,17,24,31,38$.

Evitación (EVT):

$4,11,18,25,32,39$.

Búsqueda de apoyo social (BAS):

$5,12,19,26,33,40$.

Religión (RLG):

$6,13,20,27,34,41$.

$7,14,21,28,35,42$. 